\title{
Polypharmacy and the Progression of Chronic Kidney Disease: Korean Cohort Study for Outcome in Patients with Chronic Kidney Disease
}

\author{
Hyang Ki Min ${ }^{a}$ Su Ah Sung ${ }^{a}$ Wookyung Chung ${ }^{b}$ Yeong Hoon Kim ${ }^{c}$ \\ Dong-Wan Chaed, e Curie Ahn ${ }^{d, f}$ Kook-Hwan Ohd,f Sue K. Parkg, h, i \\ Sung Woo Lee $^{j}$
}

\begin{abstract}
aDepartment of Internal Medicine, Nowon Eulji Medical Center, Eulji University, Seoul, South Korea; bepartment of Internal Medicine, Gachon University, Gil Hospital, Incheon, South Korea; 'Department of Internal Medicine, Inje University, Busan Paik Hospital, Busan, South Korea; ${ }^{d}$ Department of Internal Medicine, Seoul National University College of Medicine, Seoul, South Korea; 'Department of Internal Medicine, Seoul National University Bundang Hospital, Seongnamsi, South Korea; 'Department of Internal Medicine, Seoul National University Hospital, Seoul, South Korea; ${ }^{9}$ Department of Preventive Medicine, Seoul National University College of Medicine, Seoul, South

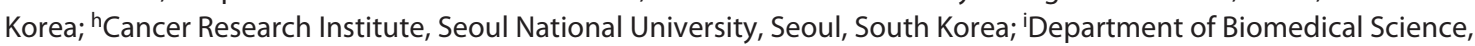
Seoul National University Graduate School, Seoul, South Korea; 'Department of Internal Medicine, Uijeongbu Eulji University Medical Center, Uijeongbu, South Korea
\end{abstract}

\section{Keywords}

Chronic kidney disease · Polypharmacy · Progression · Renal hazard · Serial medication count

\footnotetext{
Abstract

Introduction: The renal hazard of polypharmacy has never been evaluated in predialysis chronic kidney disease (CKD) patients. Objective: We aimed to analyze the renal hazard of polypharmacy in predialysis CKD patients with stage 1-5. Method: The data of 2,238 patients from a large-scale multicenter prospective Korean study (2011-2016), excluding 325 patients with various missing data, were reviewed. Polypharmacy was defined as taking 6 or more medications at the time of enrollment; renal events were defined as a $\geq 50 \%$ decrease in kidney function from baseline values, doubling of the serum creatinine levels, or initiation of renal replacement treatment. Hazard ratio (HR) and 95\% confidence interval (Cl) were calculated using Cox proportional-hazard regression analysis. Results: Of the 1,913 patients, the mean esti-
}

mated glomerular filtration rate was $53.6 \mathrm{~mL} / \mathrm{min} / 1.73 \mathrm{~m}^{2}$. The mean medication count was 4.1, and the prevalence of polypharmacy was $27.1 \%$. During the average period of 3.6 years, 520 patients developed renal events (27.2\%). Although increased medication counts were associated with increased renal hazard with HR $(95 \% \mathrm{Cl})$ of $1.056(1.007-$ $1.107, p=0.025)$, even after adjusting for various confounders, adding comorbidity score and kidney function nullified the statistical significance. In mediation analysis, 55.6\% ( $p=$ 0.016 ) of renal hazard in increased medication counts was mediated by the kidney function, and there was no direct effect of medication counts on renal event development. In subgroup analysis, the renal hazard of the medication counts was evident only in stage $1-3$ of CKD patients ( $p$ for interaction $=0.014$ ). Conclusions: We cannot identify the direct renal hazard of multiple medications, and most of the potential renal hazard was derived from intimate relationship with disease burden and kidney function.

(C) 2021 The Author(s)

Published by S. Karger AG, Basel karger@karger.com www.karger.com/kbr

Karger $\stackrel{\text { ' }}{5}$

BOPEN ACCESS
(C) 2021 The Author(s)

Published by S. Karger AG, Basel

This is an Open Access article licensed under the Creative Commons Attribution-NonCommercial-4.0 International License (CC BY-NC) (http://www.karger.com/Services/OpenAccessLicense), applicable to the online version of the article only. Usage and distribution for commercial purposes requires written permission.
Correspondence to:

Sung Woo Lee, neplsw@gmail.com 


\section{Introduction}

The prevalence of polypharmacy has been increasing worldwide [1-3]. The term polypharmacy usually refers to a condition requiring multiple medications; however, there has been no consensus on its definition. Polypharmacy is most commonly defined as regularly taking multiple medications, such as concurrently using more than 6 different drugs per day [4]. Other ways to define polypharmacy include combining the medication count and cumulative treatment duration of a drug $[2,3]$ or combining the medication count at a certain time point (e.g., during hospital stay or at discharge) $[5,6]$.

Polypharmacy has been associated with a variety of clinical outcomes. Although poor compliance may mediate the hazard of polypharmacy for mortality [7], it has been independently associated with an increased risk for all-cause and cardiovascular (CV) mortality, regardless of the compliance issue [8-10]. Furthermore, polypharmacy may be associated with an increased risk of falling [11], hip fracture [12], malnutrition [13], and impaired quality of life [14].

Patients with chronic kidney disease (CKD) often suffer from multiple comorbidities $[15,16]$; therefore, polypharmacy is inevitable and highly prevalent. In German CKD (GCKD) cohort, the prevalence of taking $\geq 5$ drugs was $81.8 \%$, which was increased with the increase of CKD stages [17]. In the CKD-Renal Epidemiology and Information Network study, the prevalence of taking $\geq 6$ drugs was $64 \%$, which was also increased with the progression of CKD [18]. In European Quality (EQUAL) study, the prevalence of taking $\geq 5$ drugs was $91.0 \%$ in CKD patients [19]. While CV medication-related polypharmacy was associated with an increased risk of acute kidney injury in elderly patients [20], psychiatric medication-related polypharmacy was associated with increased risk of future kidney disorders in patients with bipolar disorder [21]. In the community-dwelling elderly population, prescribing additional drugs had a harmful renal effect [22]; likewise, polypharmacy has the potential to deteriorate renal function. Nonetheless, there have been no studies that explore the effect of polypharmacy on the progression of CKD in predialysis CKD patients. The aim of this study was therefore to identify the effect of the medication counts and polypharmacy on the risk of CKD progression, using data of a group of adults who were enrolled in the Korean cohort study for outcome in patients with CKD (KNOWCKD).

Polypharmacy and CKD Progression

\section{Materials and Methods}

\section{Participants}

The KNOW-CKD is a multicenter, prospective cohort study in Korea consisting of 2,238 patients with predialysis CKD in stages 1-5, who were enrolled between 2011 and 2016. The detailed design and methods of the KNOW-CKD were previously published (NCT01630486 at http://www.clinicaltrials.gov) [23]. Of the 2,238 cohort subjects, 279 patients with incomplete data were excluded, including 5 with missing baseline medication counts, 56 missing data on renal events, and 264 who missed comorbidities. This study, therefore, included 1,913 patients (see online suppl. Fig. S1; see www. karger.com/doi/10.1159/000516029 for all online suppl. material).

\section{Definition of Exposure}

Data pertaining to the administrations of medications of interest (online suppl. Table S1) were collected annually. We only collected the data on prescribed medications of interests and numbers of all the prescribed medications were counted, ranged from 0 to 14. Polypharmacy was defined as a baseline medication count of $\geq 6$ [4]. Polypharmacy was used as the categorical variable and medication counts were used as the continuous variable.

\section{Definitions of Study Outcomes}

The primary outcomes were renal events, defined by halving of the estimated glomerular filtration rate (eGFR) from the baseline values; a doubling of the serum creatinine levels; and initiation of the dialysis or kidney transplantation, which were detected and annually adjudicated by both researchers and an adjudication committee. Detailed information describing the outcome measurements has been described in the previously published protocol [23]. Mostly, the patients with CKD stage $\geq 3$ were under particularly close observation and had undergone the follow-up assessments at 1- to 3-month intervals according to the local policy of all the participating centers. Regardless of the study protocol, patients who reached the end points were reported by each center. The patients were monitored until dropout, death, or December 31, 2018.

\section{Other Measurement and Definitions}

Clinical data including the detailed demographic information and baseline laboratory results were extracted from an electronic data management system (PhactaX). Body mass index (BMI) was calculated as weight $(\mathrm{kg})$ per square meter of height $\left(\mathrm{m}^{2}\right)$, and eGFR was calculated using the CKD Epidemiology Collaboration equation [24]. CKD and its stages were defined using the Kidney Disease Improving Global Outcomes 2012 guidelines [25]. Previous CV disease was defined as the physician's diagnosis of coronary artery disease, cerebrovascular disease, or peripheral vascular disease at the time of enrollment. Renin-angiotensin system (RAS) inhibitors were angiotensin-converting enzyme inhibitors or angiotensin-receptor blockers. Age-adjusted Charlson comorbidity index (ACCI) scores were calculated using additional points added for the age: a score of 1 was added for each decade after 40 years [26].

\section{Statistical Analysis}

The distributions of the continuous variables were evaluated using both histograms and Q-Q plots. Two variables were not normally distributed: the high-sensitivity C-reactive protein and urine protein-to-creatinine ratio (UPCR). The normally distributed continuous variables are expressed as mean \pm standard deviation, non- 
Fig. 1. Prevalence of polypharmacy according to the status of stages of CKD. * meant $p<0.01$ when compared to Stage 1 of CKD. $\mathrm{CKD}$, chronic kidney disease.

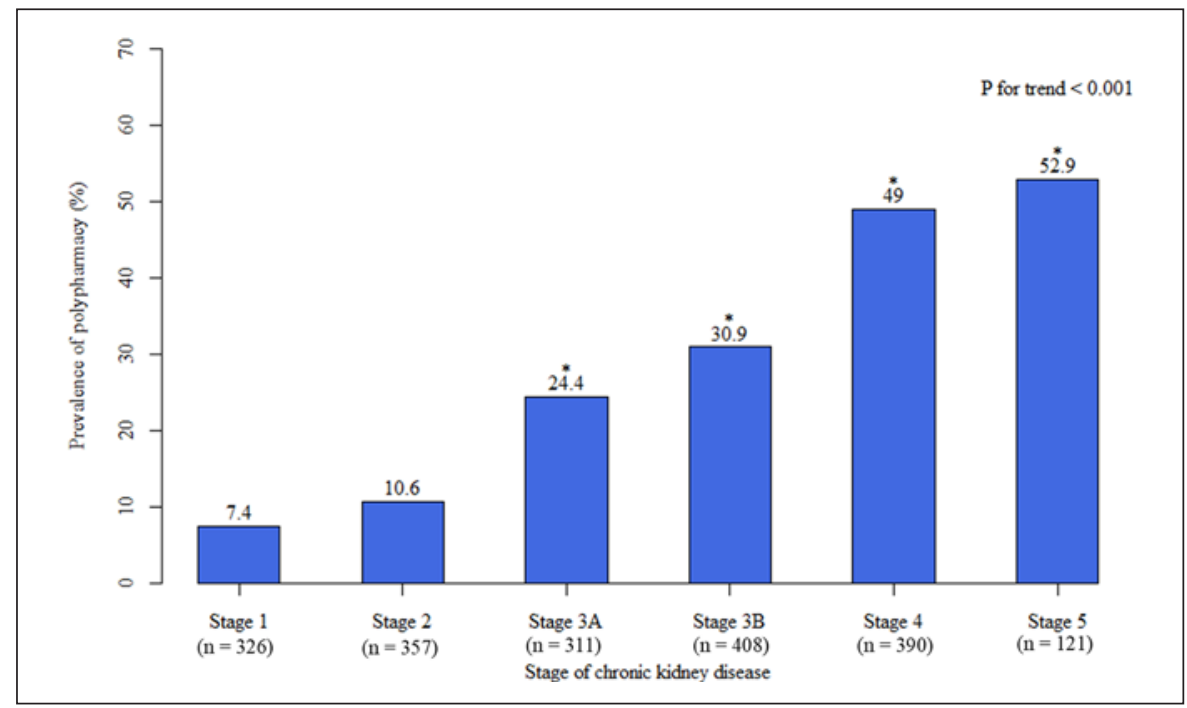

normally distributed continuous variables as medians (interquartile range), and categorical variables as percentages. Differences were analyzed for the normally distributed continuous variables using the Student's $t$-test, non-normally distributed continuous variables using the Mann-Whitney $U$ test, and categorical variables using the $\chi^{2}$ test. Kaplan-Meier curve analysis was used to calculate the renal survival, and the difference between the groups was compared via a log-rank test. The cumulative incidence curve for renal events was analyzed using the all-cause death as a competing risk, with the difference between the groups being compared using Gray's test with "cmprsk" package [27]. Hazard ratios and 95\% confidence intervals (CIs) were calculated using Cox proportionalhazard regression analyses, and proportional-hazard assumptions were tested using scaled Schoenfeld residuals, as well as the Grambsch-Therneau test with "prodlim" package [28]. In multivariate analyses, the following covariates were chosen based on the clinical and statistical relevance: age, sex, current smoking, cause of CKD, previous CV disease, RAS inhibitors, ACCI, systolic and diastolic blood pressure, BMI, fasting glucose, the blood urea nitrogen test, high-density lipoprotein cholesterol, triglyceride, white blood cell (WBC) count, hemoglobin, eGFR, and UPCR. All covariates were collected at the baseline visit. The categorical variables were sex, current smoking, and causes of CKD, previous CV disease, and RAS inhibitors. To identify the potential mediator between the exposure and outcome, causal mediation analysis was performed using 2,000 simulations with "mediation" package [29]. A $p$ value of $<0.05$ was considered statistically significant. All analyses were performed using R software Version 3.6.2 (R Foundation for Statistical Computing released 2019, Vienna, Austria).

\section{Results}

The mean age of the 1,913 patients was 53.6 years with $60.8 \%$ being men. The causes of CKD were determined to be diabetic nephropathy in $25.3 \%$ of the patients, hyper- tensive nephropathy in $19.6 \%$, glomerulonephritis in $30.7 \%$, and other causes in $24.5 \%$. The mean eGFR was $53.6 \mathrm{~mL} / \mathrm{min} / 1.73 \mathrm{~m}^{2}$, and median UPCR was $0.5 \mathrm{~g} / \mathrm{g}$ creatinine. Mean medication counts were 4.1 , and the prevalence of polypharmacy was $27.1 \%$. The prevalence of polypharmacy according to the stages of CKD increased with the progression of CKD (Fig. 1).

Baseline characteristics according to the status of polypharmacy are depicted in Table 1 . The patients with polypharmacy were often older, included a higher proportion of men, were more likely to exhibit diabetic nephropathy, and were less likely to suffer from glomerulonephritis or other causes of CKD. Similarly, the patients with polypharmacy exhibited a higher prevalence of the previous CV disease as well as a higher ACCI than those without polypharmacy; eGFR and hemoglobin levels were lower, while UPCR, WBC count, and the high-sensitivity C-reactive protein were higher in patients with polypharmacy.

In competing risk analysis, the patients with polypharmacy showed higher cumulative incidence of renal events $(p<0.001)$ than those without polypharmacy (Fig. 2). With the Grambsch-Therneau test, medication counts, age, sex, cause of CKD, previous CV disease, ACCI, RAS inhibitors, $\mathrm{BMI}$, fasting glucose, high-density lipoprotein cholesterol, WBC count, and UPCR met proportional-hazard assumption, while the smoking status, systolic and diastolic blood pressure, blood urea nitrogen test, eGFR, triglyceride, and hemoglobin did not. The potential renal hazard of polypharmacy was confirmed with univariate Cox proportional-hazard regression analysis (Table 2). The patients with polypharmacy showed increased renal hazard, with hazard ratio $(95 \% \mathrm{CI})$ of $3.023(2.541-3.598, p<0.001)$. However, 
Table 1. Baseline characteristics of patients according to polypharmacy status

\begin{tabular}{|c|c|c|c|c|}
\hline & Total $(n=1,913)$ & Non-PP $(n=1,394)$ & $\mathrm{PP}(n=519)$ & $p$ value \\
\hline Age, years & $53.6 \pm 12.3$ & $51.7 \pm 12.4$ & $58.8 \pm 10.4$ & $<0.001$ \\
\hline Male sex, $n(\%)$ & $1,164(60.8)$ & $822(59.0)$ & $342(65.9)$ & 0.007 \\
\hline Current smoking, $n(\%)$ & $308(16.1)$ & $230(16.5)$ & $78(15.0)$ & 0.479 \\
\hline Cause of CKD, $n(\%)$ & & & & $<0.001$ \\
\hline $\mathrm{DN}$ & $484(25.3)$ & $173(12.4)$ & $311(59.9)$ & $<0.001$ \\
\hline $\mathrm{HN}$ & $374(19.6)$ & $283(20.3)$ & $91(17.5)$ & 0.196 \\
\hline Others & $468(24.5)$ & $412(29.6)$ & $56(10.8)$ & $<0.001$ \\
\hline Previous CV disease, $n(\%)$ & $256(13.4)$ & $120(8.6)$ & $136(26.2)$ & $<0.001$ \\
\hline RAS inhibitor, $n(\%)$ & $1,634(85.4)$ & $1,155(82.9)$ & $479(92.3)$ & $<0.001$ \\
\hline Medication counts & $4.1 \pm 2.4$ & $2.9 \pm 1.4$ & $7.3 \pm 1.4$ & $<0.001$ \\
\hline Age-adjusted CCI & $3.4 \pm 2.2$ & $2.8 \pm 2.0$ & $5.1 \pm 1.8$ & $<0.001$ \\
\hline Systolic BP, mm Hg & $127.9 \pm 16.2$ & $126.1 \pm 15.6$ & $132.7 \pm 17.0$ & $<0.001$ \\
\hline Serum $\mathrm{Cr}, \mu \mathrm{mol} / \mathrm{L}$ & $159.6 \pm 102.4$ & $140.8 \pm 86.8$ & $210.3 \pm 122.2$ & $<0.001$ \\
\hline $\mathrm{eGFR}, \mathrm{mL} / \mathrm{min} / 1.73 \mathrm{~m}^{2}$ & $53.6 \pm 31$ & $60.1 \pm 31.5$ & $36.2 \pm 21.7$ & $<0.001$ \\
\hline Triglyceride, mmol/L & $1.8 \pm 1.1$ & $1.7 \pm 1.1$ & $1.9 \pm 1.1$ & $<0.001$ \\
\hline HDL-C, mmol/L & $1.3 \pm 0.4$ & $1.3 \pm 0.4$ & $1.2 \pm 0.4$ & $<0.001$ \\
\hline Hemoglobin, g/dL & $12.9 \pm 2$ & $13.2 \pm 1.9$ & $12.0 \pm 2.0$ & $<0.001$ \\
\hline $\mathrm{WBC}, \times 10^{3} / \mu \mathrm{L}$ & $6.6 \pm 1.9$ & $6.5 \pm 1.9$ & $6.9 \pm 2.0$ & $<0.001$ \\
\hline hsCRP, nmol/L & $5.7(1.9-15.9)$ & $5.7(1.9-14.3)$ & $7.6(2.9-19.0)$ & $<0.001$ \\
\hline UPCR, g/g Cr & $0.5(0.1-1.4)$ & $0.4(0.1-1.1)$ & $1.0(0.4-2.8)$ & $<0.001$ \\
\hline
\end{tabular}

PP, polypharmacy; CKD, chronic kidney disease; DN, diabetic nephropathy; HN, hypertensive nephropathy; GN, glomerulonephritis; CV, cardiovascular; RAS, renin-angiotensin system; CCI, Charlson Comorbidity Index; BP, blood pressure; BMI, body mass index; BUN, blood urea nitrogen; $\mathrm{Cr}$, creatinine; eGFR, estimated glomerular filtration rate; HDL-C, high-density lipoprotein cholesterol; WBC, white blood cells; hsCRP, high-sensitivity C-reactive protein; UPCR, urine protein-to-creatinine ratio. Values are expressed as mean \pm standard deviation for normally distributed continuous variables, median (interquartile range) for non-normally distributed continuous variables, and percentage for categorical variables. $p$ value was analyzed by the Student's $t$-test for normally distributed continuous variables, Mann-Whitney $U$ test for non-normally distributed continuous variables, and $\chi^{2}$ test for categorical variables.

Fig. 2. Competing the risk analysis of polypharmacy for renal events. All-cause mortality was used as competing risk.

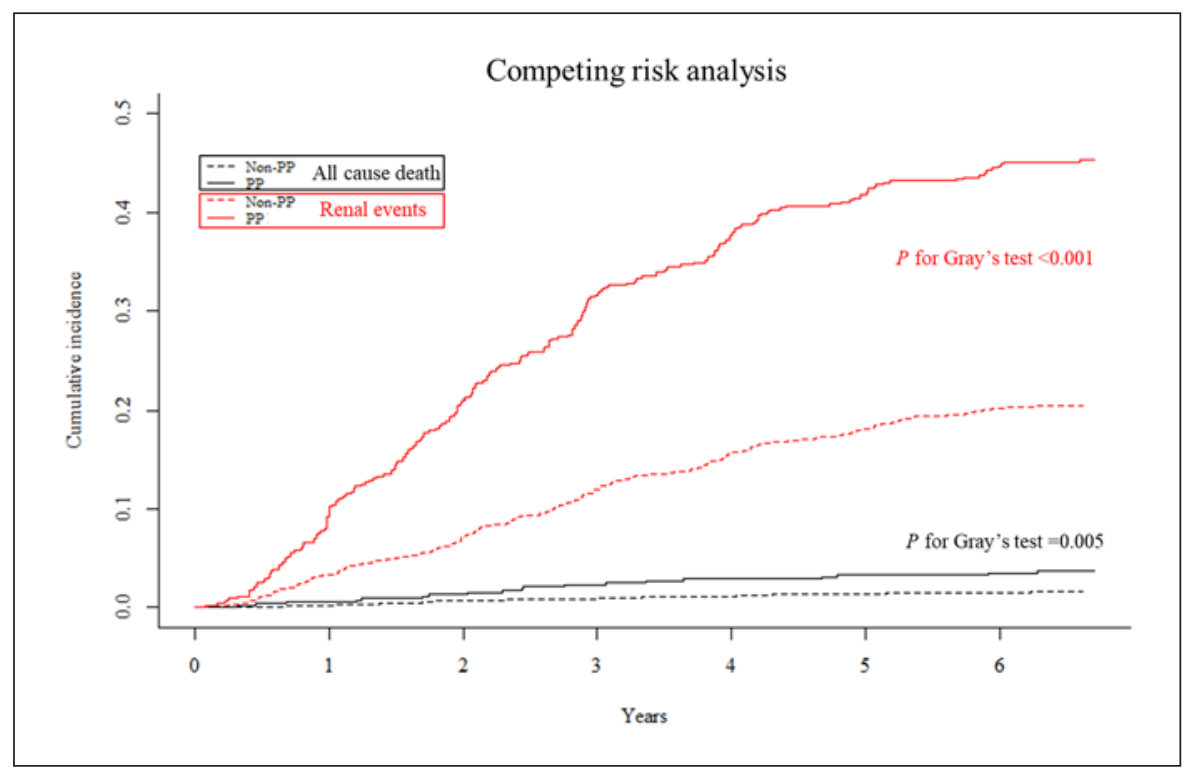


Table 2. Relationship between medication counts and renal events

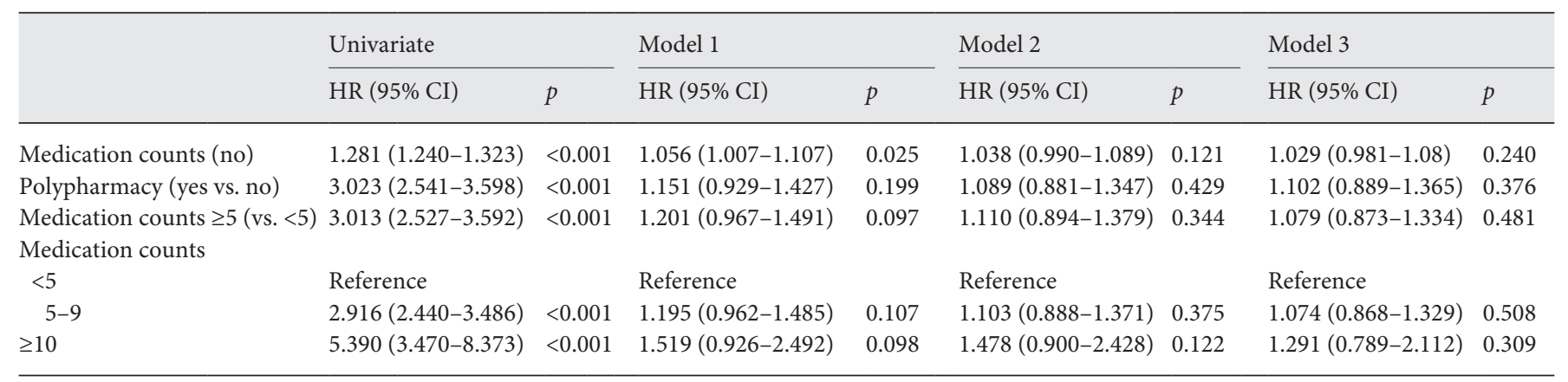

HR, hazard ratio; CI, confidence interval; CKD, chronic kidney disease; CV, cardiovascular; eGFR, estimated glomerular filtration rate; RAS, reninangiotensin system; ACCI, age-adjusted Charlson comorbidity index; UPCR, urine protein creatinine ratio; WBC, white blood cell; BUN, blood urea nitrogen; BMI, body mass index; BP, blood pressure. HR and 95\% CI were calculated using Cox proportional-hazard regression analysis. In Model 1, covariates were age, sex, current smoking, cause of CKD, previous CV disease, RAS inhibitors, systolic and diastolic BP, BMI, fasting glucose, BUN test, high-density lipoprotein cholesterol, triglyceride, WBC counts, UPCR, and hemoglobin. In Model 2, covariates were Model $1+$ ACCI. In Model 3, covariates were Model $2+$ eGFR.

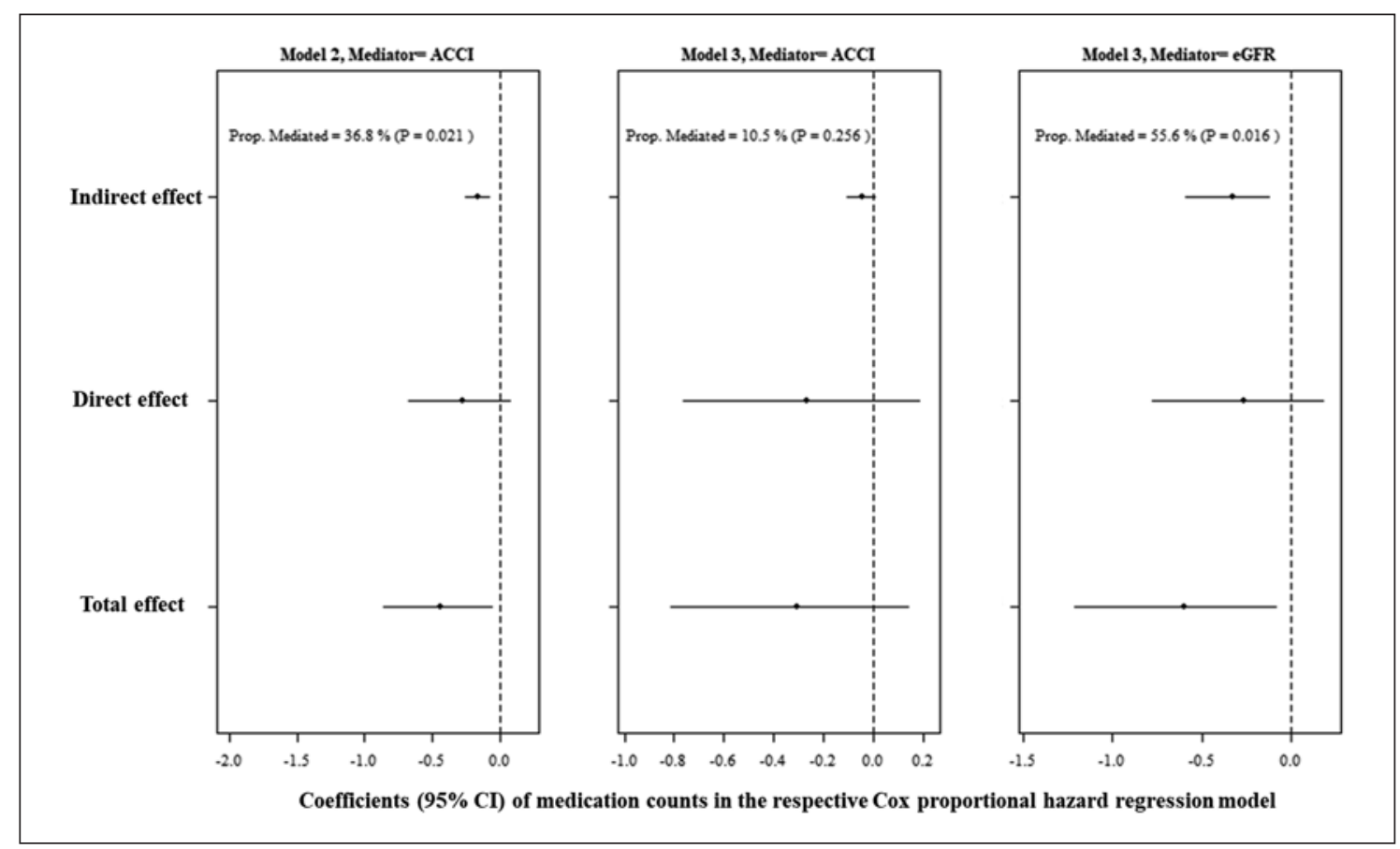

Fig. 3. Mediation analysis for the potential renal hazard of multiple medications. ACCI, age-adjusted Charlson comorbidity index; eGFR, estimated glomerular filtration rate; CI, confidence interval; Prop. Mediated, proportion mediated.

adding various confounders nullified the statistical significance of polypharmacy. In sensitivity analysis, we analyzed other cutoff of polypharmacy $(\geq 5)$ and hyperpolypharmacy $(\geq 10)$, but the results were not changed. Although the medication counts as continuous variable, maintained the statistical significance after adjusting for various con- founders (Model 1), the medication counts were not associated with renal events after adjusting for ACCI (Model 2 ), and eGFR (Model 3), suggesting potential mediating effect of ACCI and eGFR. Excluding patients with CKD stage 5 (online suppl. Table S2) and fast progressors (online suppl. Table S3) did not change the results. 
Table 3. Subgroup analysis between medication counts and renal outcome

\begin{tabular}{lllll}
\hline Subgroups & HR $(95 \% \mathrm{CI})$ & $p$ value & $\begin{array}{c}p \text { value for } \\
\text { interaction }\end{array}$ \\
\hline Age, years & $<55(n=933)$ & $1.080(1.000-1.165)$ & 0.049 & 0.401 \\
& $\geq 55(n=980)$ & $1.018(0.958-1.083)$ & 0.561 & 0.229 \\
\hline Sex & Women $(n=749)$ & $0.997(0.924-1.077)$ & 0.947 & 0.994 \\
& Men $(n=1,164)$ & $1.060(0.995-1.129)$ & 0.070 & \multirow{2}{*}{0.014} \\
\hline CKntihypertensive count & $<2(n=852)$ & $1.017(0.779-1.328)$ & 0.901 & 0.723 \\
& $\geq 2(n=1,061)$ & $1.012(0.949-1.078)$ & 0.029 & 0.713 \\
\hline
\end{tabular}

CKD, chronic kidney disease; HR, hazard ratio; CI, confidence interval; CKD, chronic kidney disease; CV, cardiovascular; eGFR, estimated glomerular filtration rate; RAS, renin-angiotensin system; ACCI, age-adjusted Charlson comorbidity index; UPCR, urine protein creatinine ratio; WBC, white blood cell; BP, blood pressure; BUN, blood urea nitrogen; BMI, body mass index. HR and 95\% CI were calculated using Cox proportional-hazard regression analysis. Covariates were age, sex, current smoking, cause of CKD, previous CV disease, RAS inhibitors, systolic and diastolic BP, BMI, fasting glucose, BUN test, high-density lipoprotein cholesterol, triglyceride, WBC counts, hemoglobin, ACCI, eGFR, and UPCR. When covariates were chosen as a subgroup, they were excluded from the model.

To identify the potential mediating effect of ACCI and eGFR, we performed the mediation analysis (Fig. 3). Using Model 2 in Table 2, increased medication counts did not show direct effect on renal events (estimate -0.275 , $95 \%$ CI -0.677 to $0.07, p=0.127)$. However, indirect effect via ACCI was statistically significant (estimate -0.161 , $95 \%$ CI -0.263 to $-0.08, p<0.001)$, with proportion mediated of $36.8 \%(p=0.021)$. However, using Model 3 in Table 2, the mediation effect of ACCI was nullified $(10.5 \%, p=0.256)$. Instead, indirect effect via eGFR was statistically significant (estimate $-0.331,95 \%$ CI -0.595 to $-0.12, p=0.001)$ with proportion mediated of $55.6 \%(p=$ $0.016)$.

In subgroup analysis (Table 3), effect modification of CKD stage on the relationship between medication counts and renal events was identified. In detail, increased medication counts were associated with the increased renal hazard, particularly in early $\mathrm{CKD}$ ( $p$ for interaction $=$ $0.014)$.

\section{Discussion}

Polypharmacy issue has been intensively studied within the elderly population [4]. The most common definition of polypharmacy is the concurrent use of $\geq 5$ or $\geq 6$ drugs [4]; the prevalence of polypharmacy using this def- inition was approximately $20-30 \%$ in the communitydwelling elderly population [30-32]. However, not only the elderly are at risk; adults with chronic diseases, such as cancer [33], chronic obstructive pulmonary disease [34], and diabetes [35] are in danger as a result of polypharmacy; patients with $\mathrm{CKD}$, a disease commonly associated with the premature aging [36], are at particular risk [17-19]. Although polypharmacy has a significant potential to deteriorate kidney function $[22,37]$, there have been no studies that explore the hazard of polypharmacy on the progression of CKD in the CKD population. In this large-scaled prospective predialysis CKD cohort, however, we could not confirm the direct effect of multiple medications on the CKD progression. Instead, the potential renal hazard of multiple medications was mediated largely by intimate relationship between the multiple medication, disease burden, and kidney function.

In this study, increased medication counts from the enrollment were associated with increased renal hazard events, adjusting for various confounders; however, adjustment for the comorbidity score nullified the renal hazard of increased medication counts. In mediation analysis, $36.8 \%$ of renal hazard of multiple medications was mediated by the relationship with the comorbidity score, and there was no direct effect of multiple medications on the CKD progression, suggesting the potential of renal hazard was a mere reflection of the disease burden. 
In this study, we did not collect all prescribed medications, and the medications of interest were not nephrotoxic, but rather nephroprotective (online suppl. Table S1). Generally, the renal hazard of polypharmacy can be caused by the increased risk of the drug-drug interactions [38], which may result in an unexpected nephrotoxicity, such as anticoagulant-related nephropathy [39]. Polypharmacy may additionally increase the risk of frailty [40], falls [11], and fracture [12], which would in turn increase the possibility of using nephrotoxins, such as nonsteroidal anti-inflammatory drugs or aminoglycoside. With the current study design, these aspects of multiple medications cannot be evaluated. Therefore, the null effect of multiple medications on the CKD progression needs to be confirmed in future studies which collect all kinds of medications including over-the-counter (OTC) medications and topical preparations.

According to the mediation analysis, adding eGFR nullified the mediation effect of ACCI and the eGFR was found to be a significant mediator between the multiple medications and CKD progression. More than half of the renal hazard of the multiple medications was attributed to the intimate relationship with kidney function. Similarly, kidney function showed a significant effect modification on the relationship between the multiple medications and CKD progression in subgroup analysis. Therefore, we concluded that along with the disease burden, intimate relationship between the multiple medications and kidney function was the fundamental mechanism of the potential renal hazard in the multiple medications in CKD patients. Since patients with early CKD were at particular risk of CKD progression associated with multiple medications, extra-cautions are needed for those patients.

The study has several limitations. First, this was an observational study; therefore, a strict causal relationship could not be ascertained using only the study results. However, the renal hazard of polypharmacy has never been suggested elsewhere, therefore, this limitation may be acceptable in this context. Second, the KNOW-CKD cohort was not primarily designed for the identification of renal hazard due to polypharmacy. Only medications of interest were collected, not all the medications were prescribed. Additionally, the data regarding supplementary OTC drugs, herb medications, and nephrotoxic drugs, including nonsteroidal anti-inflammatory drugs, were not available. These may be the reason why the prevalence of polypharmacy was low in this study. However, the incomplete collection cannot explain the low prevalence of polypharmacy in the current study entirely. In KNOW-CKD cohort, we collected almost all the major classes of the medications (online suppl. Table S1), and increased overall medication counts were largely affected by the increased medication counts of major classes [18]. Furthermore, the incomplete collection issue was not the problem of our study only. In GCKD cohort, medication counts were assessed as a patient-reported item, suggesting the possibility of under or overestimation in medication counts [17]. In EQUAL study, the medication counts were collected using the registry linkage, which was missing OTC drugs and was available only for a limited list of the prescribed medications [19]. In addition to the incomplete data collection, higher kidney function of KNOW-CKD cohort participants than the participants in GCKD, CKD-Renal Epidemiology and Information Network, and EQUAL [17-19] may also affect low prevalence of polypharmacy in this study. Third, the single ethnicity of the study population limited our ability to generalize the results. However, this study has some strength; first, the study was based on a globally recognized CKD cohort [41, 42]. Second, this was the first study to identify the potential mediators between multiple medications and the progression of CKD; this approach was possible as a result of the standardized and serial collection of data regarding the medications of interest. Third, the multicenter setting minimized the selection and center-specific bias. Finally, sufficient outcome development and fair follow-up duration strengthened our results.

\section{Conclusion}

Multiple medications did not show direct effect on the progression of CKD. Most of the potential renal hazard of multiple medications was mediated by disease burden and kidney function. Since patients with early CKD were at particular risk of CKD progression in association with multiple medications, those patients need to be monitored more cautiously for their renal health.

\section{Acknowledgements}

There are no acknowledgements to declare.

\section{Statement of Ethics}

The protocol of the KNOW-CKD adhered to the principles of the Declaration of Helsinki and was approved by the Institutional Review Board of each participating hospital, including 
Seoul National University Hospital (Approval number: 1104089-359), Yonsei University Severance Hospital (4-2011-0163), Kangbuk Samsung Medical Center (2011-01-076), Seoul St. Mary's Hospital (KC11OIMI0441), Gil Hospital (GIRBA2553), Nowon Eulji Medical Center (201105-01), Chonnam National University Hospital (CNUH-2011-092), and Busan Paik Hospital (11-091). Written informed consent was obtained from all the subjects.

\section{Conflict of Interest Statement}

The authors have no conflicts of interest to declare.

\section{Funding Sources}

This study was supported by the Research Program funded by the National Research Foundation of Korea (Grant No: NRF2016R1A6A3A11933465) and the Korea Center for Disease Control and Prevention (Grant No: 2011E3300300, 2012E3301100, 2013E3301600,2013E3301601,2013E3301602, and2016E3300200).

\section{Author Contributions}

S.W.L. - research idea and study design. S.A.S. - data acquisition. W.K.C., Y.H.K., D.W.C., C.A. and K.H.O., H.K.M., S.K.P., and S.W.L. - data analysis/interpretation. H.K.M and S.W.L. original draft. S.A.S., Y.H.K., and K.H.O. - supervision or mentorship. All the authors read and approved the final manuscript.

\section{References}

1 Kristensen RU, Norgaard A, Jensen-Dahm C, Gasse C, Wimberley T, Waldemar G. Changes in the prevalence of polypharmacy in people with and without dementia from 2000 to 2014: a nationwide study. J Alzheimers Dis. 2019;67:949-60.

2 Veehof L, Stewart R, Haaijer-Ruskamp F, Jong BM. The development of polypharmacy. A longitudinal study. Fam Pract. 2000;17: $261-7$.

3 Nishtala PS, Salahudeen MS. Temporal trends in polypharmacy and hyperpolypharmacy in older New Zealanders over a 9-year period: 2005-2013. Gerontology. 2015;61:195-202.

4 Masnoon N, Shakib S, Kalisch-Ellett L, Caughey GE. What is polypharmacy? A systematic review of definitions. BMC Geriatr. 2017;17:230.

5 Nobili A, Marengoni A, Tettamanti M, Salerno F, Pasina L, Franchi C, et al. Association between clusters of diseases and polypharmacy in hospitalized elderly patients: results from the REPOSI study. Eur J Intern Med. 2011;22:597-602.

6 Sganga F, Vetrano DL, Volpato S, Cherubini A, Ruggiero C, Corsonello A, et al. Physical performance measures and polypharmacy among hospitalized older adults: results from the CRIME study. J Nutr Health Aging. 2014 18:616-21.

7 Wu JY, Leung WY, Chang S, Lee B, Zee B, Tong PC, et al. Effectiveness of telephone counselling by a pharmacist in reducing mortality in patients receiving polypharmacy: randomised controlled trial. BMJ. 2006;333: 522.

8 Leelakanok N, Holcombe AL, Lund BC, Gu X, Schweizer ML. Association between polypharmacy and death: a systematic review and meta-analysis. J Am Pharm Assoc (2003). 2017;57:729-e10.

9 Martinez-Gomez D, Guallar-Castillon P, Higueras-Fresnillo S, Banegas JR, Sadarangani KP, Rodriguez-Artalejo F. A healthy lifestyle attenuates the effect of polypharmacy on total and cardiovascular mortality: a national prospective cohort study. Sci Rep. 2018;8:12615.

10 Porter B, Arthur A, Savva GM. How do potentially inappropriate medications and polypharmacy affect mortality in frail and nonfrail cognitively impaired older adults? A cohort study. BMJ Open. 2019;9:e026171.

11 Zia A, Kamaruzzaman SB, Tan MP. Polypharmacy and falls in older people: balancing evidence-based medicine against falls risk. Postgrad Med. 2015;127:330-7.

12 Lai SW, Liao KF, Liao CC, Muo CH, Liu CS, Sung FC. Polypharmacy correlates with increased risk for hip fracture in the elderly: a population-based study. Medicine. 2010;89:295-9.

13 Little MO. Updates in nutrition and polypharmacy. Curr Opin Clin Nutr Metab Care. 2018;21:4-9.

14 Wozniak I, Kolonko A, Chudek J, Nowak L, Farnik M, Wiecek A. Influence of polypharmacy on the quality of life in stable kidney transplant recipients. Transplant Proc. 2018; 50:1896-9.

15 Titze S, Schmid M, Kottgen A, Busch M, Floege J, Wanner C, et al. Disease burden and risk profile in referred patients with moderate chronic kidney disease: composition of the German Chronic Kidney Disease (GCKD) cohort. Nephrol Dial Transplant. 2015;30: 441-51.

16 Jhee JH, Joo YS, Park JT, Yoo TH, Park SK, Jung $\mathrm{JY}$, et al. Intensity of statin therapy and renal outcome in chronic kidney disease: results from the Korean Cohort Study for Outcome in Patients With Chronic Kidney Disease. Kidney Res Clin Pract. 2020;39:93-102.

17 Schmidt IM, Hubner S, Nadal J, Titze S, Schmid M, Barthlein B, et al. Patterns of medication use and the burden of polypharmacy in patients with chronic kidney disease: the German Chronic Kidney Disease study. Clin Kidney J. 2019;12:663-72.

18 Laville SM, Metzger M, Stengel B, Jacquelinet C, Combe C, Fouque D, et al. Chronic Kidney Disease-Renal E, Information Network Study
C: evaluation of the adequacy of drug prescriptions in patients with chronic kidney disease: results from the CKD-REIN cohort. Br J Clin Pharmacol. 2018;84:2811-23.

19 Hayward S, Hole B, Denholm R, Duncan P, Morris JE, Fraser SDS, et al. International prescribing patterns and polypharmacy in older people with advanced chronic kidney disease: results from the European Quality study. Nephrol Dial Transplant. 2020 Jun 16;36: 503-11.

20 Chao CT, Tsai HB, Wu CY, Lin YF, Hsu NC, Chen JS, et al. Cumulative cardiovascular polypharmacy is associated with the risk of acute kidney injury in elderly patients. Medicine. 2015;94:e1251.

21 Nestsiarovich A, Kerner B, Mazurie AJ, Cannon DC, Hurwitz NG, Zhu Y, et al. Comparison of 71 bipolar disorder pharmacotherapies for kidney disorder risk: the potential hazards of polypharmacy. J Affect Disord. 2019;252:201-11.

22 Ernst R, Fischer K, de Godoi Rezende Costa Molino C, Orav EJ, Theiler R, Meyer U, et al. Polypharmacy and kidney function in community-dwelling adults age 60 years and older: a prospective observational study. J Am Med Dir Assoc. 2020;21(2):254-9.

23 Oh KH, Park SK, Park HC, Chin HJ, Chae DW, Choi KH, et al. KNOW-CKD (KoreaN cohort study for Outcome in patients With Chronic Kidney Disease): design and methods. BMC Nephrol. 2014;15:80-2369.

24 Levey AS, Stevens LA, Schmid CH, Zhang YL, Castro AF 3rd, Feldman HI, et al. A new equation to estimate glomerular filtration rate. Ann Intern Med. 2009;150:604-12.

25 Stevens PE, Levin A; KDIGO Guideline Development Work Group Members. Evaluation and management of chronic kidney disease: synopsis of the kidney disease: improving global outcomes 2012 clinical practice guideline. Ann Intern Med. 2013;158:825-30.

26 Charlson M, Szatrowski TP, Peterson J, Gold $\mathrm{J}$. Validation of a combined comorbidity index. J Clin Epidemiol. 1994;47:1245-51. 
27 Gray B. cmprsk: subdistribution analysis of competing risks. R package version -. 2019. Available from: https://CRANR-projectorg/ package $=$ cmprsk.

28 Gerds TA. prodlim: product-limit estimation for censored event history analysis. R package version . 2019. Available from: https:// CRANR-projectorg $/$ package $=$ prodlim .

29 Tingley D, Yamamoto T, Hirose T, Keele L, Imai K. mediation: R package for causal mediation analysis. J Statist Soft. 2014;59:1-38.

30 Carmona-Torres JM, Cobo-Cuenca AI, Recio-Andrade B, Laredo-Aguilera JA, Martins MM, Rodriguez-Borrego MA. Prevalence and factors associated with polypharmacy in the older people: 2006-2014. J Clin Nurs. 2018; 27:2942-52.

31 Gutierrez-Valencia M, Aldaz Herce P, Lacalle-Fabo E, Contreras Escamez B, Cedeno-Veloz B, Martinez-Velilla N. Prevalence of polypharmacy and associated factors in older adults in Spain: data from the National Health Survey 2017. Med Clin. 2019;153:141-50.

32 Junius-Walker U, Theile G, Hummers-Pradier E. Prevalence and predictors of polypharmacy among older primary care patients in Germany. Fam Pract. 2007;24:14-9.
33 Kierner KA, Weixler D, Masel EK, Gartner V, Watzke HH. Polypharmacy in the terminal stage of cancer. Support Care Cancer. 2016; 24:2067-74.

34 Hanlon P, Nicholl BI, Jani BD, McQueenie R, Lee D, Gallacher KI, et al. Examining patterns of multimorbidity, polypharmacy and risk of adverse drug reactions in chronic obstructive pulmonary disease: a cross-sectional UK Biobank study. BMJ Open. 2018;8:e018404.

35 Li J, Chattopadhyay K, Xu M, Chen Y, Hu F, Wang X, et al. Prevalence and predictors of polypharmacy prescription among type 2 diabetes patients at a tertiary care department in Ningbo, China: a retrospective database study. PLoS One. 2019;14:e0220047.

36 Kooman JP, Dekker MJ, Usvyat LA, Kotanko $\mathrm{P}$, van der Sande FM, Schalkwijk CG, et al. Inflammation and premature aging in advanced chronic kidney disease. Am J Physiol Renal Physiol. 2017;313:F938-50.

37 Kang H, Hong SH. Risk of kidney dysfunction from polypharmacy among older patients: a nested case-control study of the South Korean senior cohort. Sci Rep. 2019;9:10440.
38 Nusair MB, Al-Azzam SI, Arabyat RM, Amawi HA, Alzoubi KH, Rabah AA. The prevalence and severity of potential drugdrug interactions among adult polypharmacy patients at outpatient clinics in Jordan. Saudi Pharm J. 2020;28:155-60.

39 Brodsky S, Eikelboom J, Hebert LA. Anticoagulant-related nephropathy. J Am Soc Nephrol. 2018;29(12):2787-93.

40 Mach J, Gemikonakli G, Logan C, Vander Wyk B, Allore H, Ekambareshwar S, et al. Chronic polypharmacy with increasing Drug Burden Index (DBI) exacerbates frailty and impairs physical function, with effects attenuated by deprescribing, in aged mice. J Gerontol A Biol Sci Med Sci. 2020 Mar 9:glaa060. Epub ahead of print.

41 Orlandi PF, Huang J, Fukagawa M, Hoy W, Jha $\mathrm{V}$, Oh $\mathrm{KH}$, et al. A collaborative, individual-level analysis compared longitudinal outcomes across the International Network of Chronic Kidney Disease (iNETCKD) cohorts. Kidney Int. 2019;96:1217-33.

42 Alencar de Pinho N, Levin A, Fukagawa M, Hoy WE, Pecoits-Filho R, Reichel H, et al. Considerable international variation exists in blood pressure control and antihypertensive prescription patterns in chronic kidney disease. Kidney Int. 2019;96:983-94. 